\title{
A GERONTOLOGIA INTERFACE O MEIO AMBIENTE COMO ESTRATÉGIA NO CUIDADO E PROMOÇÃO DA SAÚDE
}

\author{
Paulo Barrozo Cassol
}

\section{RESUMO}

A transição demográfica e epidemiológica mundial está resultando no envelhecimento populacional, esse fenômeno encontra-se em diferentes fases no mundo, exigindo uma reorganização do sistema de Saúde. O envelhecimento populacional no Brasil cresce em números, aproximando a valores dos países desenvolvidos, no entanto a questão social não acompanha essa trajetória. Este estudo tem por objetivo estimular a discussão para a necessidade de políticas públicas de cuidado ao idoso com a Interface Saúde e Meio Ambiente como estratégia no cuidado e promoção da saúde. Trata-se de um estudo teórico embasado em literatura especializada. Concluímos que a promoção da saúde envolve os apoios educacionais e ambientais. A interdisciplinaridade, dos cursos de saúde com os das áreas ambientais são um dos caminhos para desenvolvimento de propostas inovadoras na busca de soluções e na promoção da saúde ao idoso interface meio ambiente. $O$ meio Ambiente e saúde são indissociáveis, nesse processo as relações entre os homens e o ambiente podem ser favoráveis ou prejudiciais à saúde, portanto a sua compressão é fundamental para a promoção da saúde.

Palavras-chave: Educação Ambiental; Interdisciplinaridade; Meio Ambiente; Saúde.

\begin{abstract}
The demographic and epidemiological transition is resulting in global population aging, this phenomenon is at different stages in the world, requiring a reorganization of Health Population aging in Brazil is growing in numbers, approaching the values of developed countries, however the question social does not follow this path. This study aims to stimulate discussion of the need for public policies on elderly care interface with the Health and Environment as a strategy for care and health promotion. It is grounded in a theoretical study of literature. We conclude that health promotion involves the educational and environmental support. The interdisciplinarity of courses with the health of natural areas is one way to develop innovative approaches to finding solutions and promoting health for the elderly interface environment. The Environment and health are inextricably linked, in that case the relationship between humans and the environment may be favorable or detrimental to health, so your compression is essential for health promotion
\end{abstract}

Keywords: Environmental Education; Interdisciplinarity; Environment; Health.

\section{INTRODUÇÃO}

Com o crescente envelhecimento populacional mundial, temos um aumento da prevalência de doenças físicas, mentais e sociais, com intenso comprometimento na qualidade de vida desses indivíduos. Quando se pensa em saúde, em gerontologia é importante destacarmos as questões ambientais por sua reflexibilidade na saúde física e mental assim como o entendimento dessas relações frente a esse fenômeno (VERAS, 2009; BAGGIO; CALLEGARO; ERDMANN, 2011). O meio 
Ambiente e saúde são indissociáveis, nesse processo as relações entre os homens e o ambiente podem ser favoráveis ou prejudiciais à saúde, portanto a sua compressão é fundamental para a promoção da saúde.

A transição demográfica e epidemiológica mundial esta resultando no envelhecimento populacional, esse fenômeno encontra-se em diferentes fases no mundo exigindo uma reorganização do sistema de Saúde, devido às doenças crônicas dessa população. O crescente envelhecimento nos países da América Latina, especialmente o Brasil, é semelhante ao ocorrido na Europa. Entretanto existe um grande diferencial nesse processo de transição demografia, no modelo Europeu ocorreu desenvolvimento social e aumento de renda. Enquanto que na América Latina ocorreu um processo de urbanização sem alteração da distribuição de renda, ou seja, os ganhos sociais não acompanharam esse processo (NASRI, 2008).

O presente artigo visa a estimular a discussão para a necessidade de políticas públicas de cuidado ao idoso que contemplem a Interface: Gerontologia, Saúde e Meio Ambiente como estratégia no cuidado e promoção da saúde. Trata-se de um estudo teórico reflexivo embasado em literatura especializada, como meio de discussão diante desse cenário do aumento da população idosa no Brasil. O tema abordado é de fundamental importância para os gestores e profissionais de saúde, como forma de melhoria na qualidade de vida dessa população, assim como a busca de estratégias inovadoras na promoção da saúde do idoso.

\section{O MEIO AMBIENTE E A SUA REFLEXIBILIDADE NA SAÚDE}

Ao pensarmos em determinantes de saúde, nos remete a Lei 8.080, de 19 de setembro de 1990, a qual preconiza que a saúde tem como fatores determinantes e condicionantes a alimentação, a moradia, o saneamento básico, o meio ambiente, o trabalho, a renda, a educação, o transporte, o lazer e também o acesso aos bens e serviços essenciais; e que os níveis de saúde da população são um meio de demonstrar como está o nível da organização social e econômica do País (BRASIL, 2006).

Estudos apontam uma melhor condição na saúde da população, por meio de melhorias na educação, no transporte, na coleta e no destino dos resíduos, na cultura, no esporte, no lazer, na promoção da qualidade ambiental entre outros. Sendo que esses resultados são muito mais intensos e duráveis do que aqueles proporcionados pelos serviços assistenciais (BRASIL, 2007).

O perfil da saúde da população brasileira na atualidade é formado por três cenários, os quais são condicionados por diferentes contextos socioambientais. O primeiro cenário ocorre predominantemente às doenças cardiovasculares e neoplásicas, cuja tendência crescente nos últimos dez anos acompanha o envelhecimento da população, com o efeito de condições genéticas, de vida e trabalho, principalmente por exposições a determinados poluentes ambientais. No segundo cenário, é formado pelas doenças infecto-parasitárias, claramente determinadas pelas condições socioambientais. Enquanto no terceiro cenário, temos as situações que engloba os acidentes e as violências. Estes três cenários evidenciam os acontecimentos socioambientais como produtores de traumas, lesões e doenças, ou seja, o perfil da saúde da população brasileira. Neste contexto a Declaração de Alma-Ata para os Cuidados Primários em Saúde de 1978, trouxe um novo pensamento na caracterização do processo saúde-doença, incluindo nesse processo as dimensões sociais, políticas, culturais, ambientais e econômicas como componentes necessários nas ações e aos serviços de saúde (BRASIL, 2007). 


\section{O ENVELHECIMENTO POPULACIONAL E A PROMOÇÃO DA SAÚDE}

Gerontologia é o estudo dos fenômenos que afetam a velhice; nos aspectos morfológicos, fisiopatológicos, patológicos, geriátrico, psicológicos, sociais entre outros. O Meio ambiente é o meio em que se vive o que está em volta da pessoa ou objeto, a localidade onde se reside. Envolve as dimensões físicas, social, cultural, a econômica e a política, sejam elas familiares, de trabalho, lazer, educação, consumo, produção entre outros (Dicionário online de português; BRASIL, 2007).

$\mathrm{Na}$ atualidade vivenciamos um grande aumento de indivíduos idosos. $\mathrm{O}$ fator que caracteriza este fenômeno de envelhecimento da população não é o declínio da mortalidade, mas sim o declínio da fecundidade, na qual aumenta em números os indivíduos idosos e diminui os mais jovens ( RAMOS; VERAS; KALACHE,1987).

No Brasil a população de idosos está numa curva ascendente. Em mil novecentos e sessenta a população de idosos era três milhões, atingindo o numero de vinte milhões em dois mil e oito. Em menos de cinqüenta anos essa população teve quase $700 \%$ de aumento. Esse novo cenário brasileiro tem um impacto direto na questão da saúde, onde surge um quadro de enfermidades complexas caracterizado por doenças crônicas e múltiplas, medicação contínua e exames periódicos( VERAS,2009).

O Instituto Brasileiro de Geografia e Estatística apontam os dados do censo de 2000, no qual a população brasileira com 60 anos ou mais corresponde a $8,6 \%$ da população, cerca de quatorze milhões. As Projeções demográficas mostram que este número poderá ultrapassar os trinta milhões em apenas 25 anos. Por essa realidade o Brasil elaborou a lei n. 0 10.741, de 1. de outubro de 2003, a qual dispõe sobre o Estatuto do Idoso regulando os direitos as pessoas com idade igual ou superior a sessenta anos ( BRASIL, 2007 ).

Pelas projeções do Instituto Brasileiro de Geografia e Estatística no ano de 2030 o número de idosos será em cerca de quatro milhões a mais que o numero de crianças e adolescentes menores de 15 anos. (Brasil, 2009).

A realidade Brasileira apresenta muitos contrastes, embora em números a população idosa caminha para os padrões de países desenvolvidos, a questão social não acompanha essa trajetória. Ao depararmos com essa realidade. Qual é a relação dos profissionais de saúde com essa questão? Qual a compressão do cenário em que esta população de idosos vive e a sua reflexibilidade na saúde física e mental? Essas ponderações são importantes para uma intervenção com qualidade na assistência assim como a busca de estratégias na promoção da saúde.

Nesse sentido a promoção da saúde é uma ferramenta eficaz, conforme a primeira Conferência Internacional sobre Promoção da Saúde que apresentou a Carta de Ottawa em 1986 sendo um marco histórico no sentido da saúde, abrindo novas possibilidades. Pelo qual a carta de Ottawa apresenta o processo de promoção à saúde, que envolve a capacitação da comunidade para atuar na melhoria da sua qualidade de vida e saúde, participando em seu controle. Para atingir um completo bem-estar físico, mental e social, os indivíduos e grupos devem saber identificar aspirações, satisfazer necessidades e modificar favoravelmente o meio ambiente. Outros elementos importantes nesse processo são pré-requisitos para a saúde como: paz, habitação, educação, alimentação, renda, ecossistema estável, recursos sustentáveis, justiça social e eqüidade (BRASIL, 2002).

A Carta de Ottawa evidenciou passos importantes na promoção à saúde onde o meio ambiente é elemento fundamental na questão da saúde. Entretanto qual o significado, a compressão que o profissional de saúde tem do meio ambiente e como ele relaciona isso com a questão da saúde? 0 meio ambiente saudável envolve muito mais que o acesso aos serviços básicos como o 
fornecimento de água tratada, coleta de esgoto e de lixo, o tema é complexo, pois envolve todo um contexto social. Se sofre de violência física ou psíquica, possui relações afetivas, tem estímulos a desenvolver alguma atividade de lazer, possui segurança alimentar e moradia, acesso aos fármacos entre outros. As interações por profissionais da saúde e a população, são momentos importantes, onde se podem estabelecer relações do contexto de onde e como vivem esses indivíduos, produzindo assim uma ação efetiva no seu tratamento, pois o meio ambiente pode refletir na saúde física e mental. Nesse sentido a Educação Ambiental é uma importante ferramenta.

A promoção da saúde envolve os apoios educacionais e ambientais, mesclando os determinantes de saúde como os fatores genéticos, ambientais, de saúde e sociais. Neste contexto, a educação ambiental envolve as circunstâncias sociais, políticas e econômicas onde os fatores ambientais também são considerados quando se planeja atividades de promoção a saúde (CANDEIAS, 1997).

A Educação Ambiental é um processo, onde as pessoas participam ativamente no diagnóstico dos problemas e busca de soluções, são agentes transformadores, desenvolvendo habilidades e atitudes, por meio de uma conduta ética e condizente ao exercício da cidadania (RUIZ; LEITE; AGUIAR, 2005).

A Educação Ambiental é um processo de transformação, um dos caminhos para a preservação da biosfera, onde as pessoas participam no diagnóstico dos problemas e na busca de soluções, portanto inserida perfeitamente nesta era globalizada (CASSOL, 2012).

O desenvolvimento da interface entre saúde e ambiente, com o intuito de implementar uma política de saúde ambiental, emerge da necessidade de aprimorar o atual modelo do Sistema Único de Saúde (SUS), de forma que a promoção da saúde seja realizada, não exclusivamente nas unidades de saúde, ocorrendo também nos espaços do cotidiano da vida humana, na dinâmica da vida das cidades e do campo. Compreendendo o ambiente como um território vivo, com reflexos de processos políticos, históricos, econômicos, sociais e culturais. Nesse sentido é necessário práticas de saúde voltada para os determinantes e condicionantes da saúde (BRASIL, 2007).

Cuidado em enfermagem também está relacionado com o cuidado do ecossistema, no sentido em que se busca preservar a vida do homem e da natureza. Os problemas ambientais podem refletir em problemas de saúde. Nesse sentido é possível estabelecer uma relação complexa de cuidados, por meio de uma visão integradora entre homem-natureza, saúde ambiente (BAGGIO; CALLEGARO; ERDMANN, 2011).

É importante o profissional de saúde ser capacitado para perceber a multicausalidade dos processos mórbidos, que podem ser físicos, mentais ou sociais e contextualizando o indivíduo em seu meio ambiente. Neste contexto é uma estratégia a ser utilizado na Unidade Básica de Saúde, por meio de uma visão sistêmica e integral do indivíduo e da família e do meio onde reside o individuo, onde possa compreender os fatores que alteram o equilíbrio entre o indivíduo e o ambiente. Para tanto, é importante conhecer a realidade das famílias, seus aspectos físicos e mentais, demográficos e sociais (SILVESTRE; NETO, 2003).

Na saúde, as abordagens ecológicas datam do final da década de 1970, onde ambientalistas, sanitaristas, investigadores e gestores, começaram a perceber a necessidade de integrar suas ações, desta forma, resultaria em uma melhor qualidade de vida da população. A compressão das atividades humanas e seu reflexo no ambiente e na saúde, decorre de conhecimentos disciplinares e praticas setoriais direcionadas para uma abordagem transdiciplinar (MINAYO; MIRANDA, 2002).

As universidades são os grandes pólos geradores de conhecimento, os projetos de extensão, são uma das formas de levar o conhecimento e a capacitação para os profissionais das unidades básicas de saúde. Que poderá ser ampliado e envolver a capacitação da comunidade para atuar na melhoria da sua qualidade de vida e modificar favoravelmente o meio ambiente. Podemos incluir 
nesse processo a questão da sustentabilidade, o uso racional de energia elétrica e consumo de água, a segregação e o descarte do lixo, assim como práticas de reutilização e a reciclagem de materiais, dessa forma minimizando o impacto ambiental.

\section{CONCLUSÕES}

O fenômeno do envelhecimento populacional no Brasil cresce em números, aproximando a valores dos países desenvolvidos, no entanto a questão social não acompanha essa trajetória. 0 atendimento a essa crescente população idosa, constitui um grande desafio para o sistema de saúde. Neste contexto as universidades têm muito a contribuir, trabalhando a interface saúde do idoso e o meio ambiente tanto a nível acadêmico, como em projetos de extensão e em capacitação para as unidades básicas de saúde. Nesse sentido a saúde coletiva tem um amplo campo para desenvolver essa atividade.

As universidades são os grandes pólos geradores de conhecimento, a interdisciplinaridade dos cursos de saúde com os das áreas ambientais são um meio para desenvolvimento de propostas inovadoras na busca de soluções para promoção da saúde interface meio ambiente. A Educação Ambiental pode contribuir nesse processo, onde as pessoas participam na busca de soluções. $O$ ambiente favorável à saúde implica em menos custos para o Sistema Único de Saúde. Portanto a promoção da saúde envolve os apoios educacionais e ambientais, gerando conhecimentos e atualizações, resultando em uma melhoria na qualidade de vida para a população, com reflexibilidade no meio ambiente.

\section{REFERENCIAS BIBLIOGRÁFICAS}

BRASIL, 2002 Ministério da Saúde. Disponível em >

http://bvsms.saude.gov.br/bvs/publicacoes/cartas promocao.pdf. Acesso em 20/10/11

BRASIL, 2006. MINISTÉRIO DA SAÚDE. Disponível em

http://conselho.saude.gov.br/biblioteca/livros/coletanea miolo.pdf. Acesso em 30/09/11

BRASIL, 2007._Brasil. Ministério da Saúde. Conselho Nacional de Saúde.

Subsídios para construção da Política Nacional de Saúde Ambiental / Ministério da Saúde, Conselho Nacional de Saúde. - Brasília: Editora do Ministério da Saúde, 2007. Disponível em >

http://conselho.saude.gov.br/biblioteca/livros/subsi miolo.pdf. Acesso em 11/10/11

BRASIL, 2007. Ministério da Saúde. Estatuto do Idoso / Ministério da Saúde. - 2. Ed. rev. - Brasília: Editora do Ministério da Saúde, 2007. Disponível em >

http://conselho.saude.gov.br/biblioteca/livros/estatuto idoso2edicao.pdf. Acesso em 12/10/11

BRASIL, 2009. Instituto Brasileiro de Geografia e Estatística - IBGE. Indicadores Sociodemográficos e de Saúde. Disponível em:

http://www.ibge.gov.br/home/estatistica/populacao/indic sociosaude/2009/indicsaude.pdf.http://www.ib ge.gov.br/home/est.atistica/populacao/indic sociosaude/2009/indicsaude.pdf. Acesso em 22/10/11 Baggio, M.A.; Callegaro, G.D.; Lorenzini , Erdmann.A.L.L. reme - Rev. Min. Enferm.;15(1): 11-18, jan./mar., 2011. SIGNIFICANDO O CUIDADO ECOLÓGICO /PLANETÁRIO /COLETIVO/DOAMBIENTE À LUZ DO PENSAMENTO COMPLEXO. Disponível em http://www.enf.ufmg.br/site novo/modules/mastop publish/files/files 4e1dbbb6670cc.pdf.Acesso em $\underline{28 / 10 / 11}$ 


\section{REGETHFSM}

Rev. Elet. em Gestão, Educação e Tecnologia Ambiental

(e-ISSN: 2236-1170)

Candeias, Nelly M.F. Conceitos de educação e de promoção em saúde: mudanças individuais e mudanças organizacionais. Rev. Saúde Pública vol. 31 no. 2. São Paulo Apr. 1997doi:

10.1590/S003489101997000200016.Disponível em:

http://www.scielo.br/scielo.php?script=sci_arttext\&pid=S0034-89101997000200016.Acesso em:14/02/12.

Cassol. O VERDE ABALOU O MURO. Revista Eletrônica em Gestão, Educação e Tecnologia Ambiental. Vol. 5, No 5. p.767-772 (2012). Disponível em: http://cascavel.ufsm.br/revistas/ojs-

2.2.2/index.php/reget/article/viewFile/4211/2805. Acesso em 01.03.12.

Dicionário online de português. Disponível em> http://www.dicio.com.br/gerontologia//.acesso em 30/09/11

Dicionário online de português. Disponível em http http://www.dicio.com.br/ambiente/. Acesso em 30/09/11

Fabio Nasri. O envelhecimento populacional no Brasil. Einstein. 2008; 6 (Supl 1):S4-S6.Disponível em > http://apps.einstein.br/revista/arquivos/PDF/833-Einstein\%20Suplemento\%20v6n1\%20pS4-6.pdf. Acesso em $15 / 10 / 11$

Luiz Roberto Ramos, Renato P. Veras,Alexandre Kalache. Envelhecimento populacional: uma realidade brasileira. Rev.Saúde Publica. 1987;21(3):211-24. Disponível em > http://www.scielo.br/pdf/rsp/v21n3/06.pdf. Acesso em 20/10/11 MINAYO, M.C.S; MIRANDA,A.C. Saúde e ambiente sustentável: estreitando nós. Rio de Janeiro: Fiocruz, 2002.

Renato Veras. Envelhecimento populacional contemporâneo: demandas, desafios e inovações. Rev Saúde Pública 2009;43(3):548-54. Disponível em > http://www.saude.br/envelhecimento populacional contemporaneo demandas desafios e inovacoes 2009.pdf. Acesso em 12/10/11

RUIZ, J. B.; LEITE, E. C.; RUIZ, A. M.; AGUIAR, T. F. Educação Ambiental e os temas transversais. Revista de Ciências Humanas da UNIPAR. Akrópolis, Umuarama, v.13, no.1, jan/mar., 2005. Disponível em:

http://revistas.unipar.br/akropolis/article/view/451/410. Acesso em 30/09/11.

Silvestre, J.A.; Neto, M.M.C. Abordagem do idoso em programas de saúde da família. Cad. Saúde Pública, Rio de Janeiro, 19(3):839-847, mai-jun, 2003. Disponível em:

http://www.scielo.br/pdf/csp/v19n3/15887.pdf. Acesso em 30/10/11 